\title{
Suppression of human epidermal growth factor receptor 2 via interference increases the chemosensitivity of ovarian carcinoma
}

\author{
XIAOPING SONG ${ }^{1 *}$, KAILV SUN $^{2 *}$, JIANMING HU ${ }^{1}$ and JIANGHU ZHOU ${ }^{1}$ \\ Divisions of ${ }^{1}$ Gynecology and Obstetrics and ${ }^{2}$ General Surgery, First Affiliated Hospital of Soochow University, \\ Suzhou, Jiangsu 215006, P.R. China
}

Received March 9, 2015; Accepted December 10, 2015

DOI: $10.3892 / \mathrm{ol} .2016 .4341$

\begin{abstract}
The function of human epidermal growth factor receptor 2 (HER2) in the chemosensitivity of ovarian carcinoma has not been fully investigated, therefore, the present study aimed to analyze the potential role of HER 2 in ovarian carcinoma chemosensitivity in further detail. SKOV3 cells were transfected with lentiviral-mediated HER2-small hairpin RNA (shRNA) molecules to establish the stable expression of HER2-shRNA in the SKOV3 cell line (knockdown cells; KD) and negative control cell line (NC). The untransfected SKOV3 cell line served as the blank control $(\mathrm{CON})$ group. Reverse transcription-quantitative polymerase chain reaction (RT-qPCR) and western blot analysis were used to detect the expression of HER2 in the three different cell types, which were subsequently examined for growth inhibition using a cell counting kit- 8 assay. The CON and $\mathrm{KD}$ cell strains were utilized to establish xenograft models in nude mice, primarily to detect the expression of the HER2 protein, and additionally to observe tumor size changes under the treatment of cisplatin (DDP) chemotherapy. RT-qPCR and western blot analysis demonstrated a significant decrease in the levels of HER2 mRNA and protein in the KD cells. The suppression of HER2 expression resulted in an increase of chemotherapy sensitivity in the SKOV3 cells. HER2 protein expression decreased significantly following transduction with specific HER2-shRNA. Additionally, growth slowed significantly under treatment with DDP in ovarian cancer transplantation tumors. In conclusion, lentivirus-mediated HER2-shRNA effectively inhibits the expression of the HER2 gene, and increases the chemosensitivity to DDP in ovarian carcinoma.
\end{abstract}

Correspondence to: Professor Jianming $\mathrm{Hu}$, Division of Gynecology and Obstetrics, First Affiliated Hospital of Soochow University, 188 Shizi Street, Suzhou, Jiangsu 215006, P.R. China E-mail: hu_jianming19@163.com

*Contributed equally

Key words: ovarian carcinoma, neoplasm transplantation, RNA interference, cisplatin, chemosensitivity

\section{Introduction}

Ovarian carcinoma is a lethal gynecological malignancy with a 5-year survival rate of $25-30 \%$ (1). At present, $60 \%$ of advanced-stage patients will experience disease recurrence (2), whilst the average life expectancy is 12 to 18 months (3). The primary treatment for ovarian cancer is cytoreductive surgery and adjuvant platinum-based chemotherapy (3). The low ovarian carcinoma survival rates are known to be largely impacted by the development of chemotherapy resistance (4), with improved treatments now required for patients.

Human epidermal growth factor receptor 2 (HER2), a member of the epidermal growth factor receptor (EGFR) family, is located on the chromosome 17 q21 band. As a key gene involved in cell survival, differentiation, proliferation, apoptosis and migration, HER2 gene amplification and protein overexpression may result in malignant transformation (5-8). The present study demonstrates that the HER2 gene is highly expressed in ovarian carcinoma and is closely associated with cancer cell growth, as well as differentiation, chemical resistance, malignant transformation and patient prognosis (9-12). RNA interference (RNAi) technology is now recognized as a common research tool for the silencing of target genes (13), and as a revolutionary class of therapeutics for the treatment of cancer (14). RNAi may be activated by double-stranded RNAs, which are processed into small fragments (21-23 base pairs) by the Dicer enzyme (15);these fragments are then loaded into the RNA-induced silencing complex (RISC). Following this, the activated RISC-guide strand complex is directed to the target mRNA complementary region, which is then degraded and therefore inhibited from being translated (16). A previous study demonstrated that the transfection of HER2 small interfering RNA (siRNA) into SKOV3 cells induced apoptosis, and inhibited proliferation and the invasive and migratory phenotypes of the SKOV3 cells (4).

In the present study, RNAi technology was utilized to design siRNAs that targeted different regions within the open reading frame of HER2 mRNA, in order to examine the chemosensitivity of ovarian carcinoma.

\section{Materials and methods}

Cell lines and culture. The ovarian cancer SKOV 3 cell line was obtained from the Jiangsu Institute of Hematology (Suzhou, 
China). Cell culture was performed according to the manufacturer's protocols. The cells were routinely maintained in RPMI 1640 Medium (HyClone; GE Healthcare Life Sciences, Logan, UT, USA), supplemented with $10 \%$ fetal bovine serum (HyClone; GE Healthcare Life Sciences), $1 \%$ penicillin and $1 \%$ streptomycin in a well-humidified incubator with $5 \% \mathrm{CO}_{2}$ at $37^{\circ} \mathrm{C}$. SKOV3 cells were transfected with lentiviral-mediated HER2-small hairpin RNA (shRNA) molecules to establish the stable expression of HER2-shRNA in the SKOV3 cell line (knockdown cells; KD) and negative control cell line (NC). The untransfected SKOV3 cell line served as the blank control (CON) group.

siRNA preparation and infection. HER 2 cDNA sequences (NM_004448) were selected based on HER2 target sites (obtained from GenBank; www.ncbi.nlm.nih.gov/genbank) using the BLOCK-iT ${ }^{\mathrm{TM}}$ RNAi Designer (Invitrogen; Thermo Fisher Scientific, Inc., Waltham, MA, USA). siRNA design principles described by Tuschl (17) were used for the design of the three target sequences of RNAi and to aid the selection of a negative control (NC) sequence. In previous experiments, the present study designed three target sequences of RNAi and an NC sequence: HER2-RNAi-1, sense TCTGCGGTGGTTGGC ATTC (2204-2222; 57.89\% GC); HER-2-RNAi-1, sense ATA TGTGAACCAGCCAGAT (3652-3670; 42.11\% GC); HER2RNAi-3, sense GTGCCAATATCCAGGAGTT (1311-1329; 47.37\% GC); and HER2-RNAi-NC, sense TTCTCCGAACGT GTCACGT. Following screening the most effective interference sequence was HER2-RNAi-1 sense. Using Lipofectamine $^{\circledR} 2000$ (Thermo Fisher Scientific, Inc.), the siRNAs were transfected into $293 \mathrm{~T}$ cells, and viral supernatants were harvested and infected into the human ovarian cancer SKOV3 cell line. Following a total of $12 \mathrm{~h}$ post-infection, the medium was replaced with medium containing puromycin $(1.5 \mu \mathrm{g} / \mathrm{ml})$, and 2 weeks later the medium was replaced by normal medium and cells were cultured to expand.

Reverse transcription-quantitative polymerase chain reaction (RT-qPCR) analysis. Total RNA was extracted using TRIzol ${ }^{\circledR}$ (Invitrogen; Thermo Fisher Scientific, Inc.) according to the manufacturer's protocols, and was stored at $-80^{\circ} \mathrm{C}$. The RNA concentration and purity was measured using a spectrophotometer (BioPhotometer Plus; Eppendorf, Hamburg, Germany). According to the Transcriptor One-Step RT-PCR kit (Roche Diagnostics, Basel, Switzerland) protocols, $1 \mu \mathrm{g}$ total RNA (Promega Corporation, Madison, WI, USA) was reverse-transcribed. Reverse transcriptase was purchased from Roche Diagnostics, (Basel, Switzerland). $\beta$-actin was used as an internal reference. DNA polymerase/DNase was purchased from Promega Corp. (Madison, WI, USA). The primer sequences (Shanghai Sangon Biological Engineering Technology Services Ltd., Shanghai, China) used were as follows: Forward, 5'-CTGAACAATACCACCCCTGTC-3'; and reverse, 5'-AGATGTCCTTCCACAAAATCGT-3'. The reaction conditions were a two-step procedure hot-start activation at $95^{\circ} \mathrm{C}$ for $2 \mathrm{~min}$ and 40 cycles, with denaturation at $95^{\circ} \mathrm{C}$ for $15 \mathrm{sec}$, followed by annealing/elongation at $60^{\circ} \mathrm{C}$ for $60 \mathrm{sec}$ and finally dissociation at $60-95^{\circ} \mathrm{C}$. Abosorbance values were read and the $2^{-\Delta \Delta \mathrm{Cq}}$ was used to calculate target gene expression. PCR was performed on the ABI 7500 PCR System (Applied Biosystems;
Thermo Fisher Scientific, Inc.). Furthermore, each sample was subjected to RT-qPCR testing using the same conditions in triplicate, and the average values were used for data analysis.

Western blot analysis. Extraction of total protein from the cells was performed using radioimmunoprecipitation assay buffer and protein quantification was conducted using Coomassie brilliant blue stain. A total of $50 \mu \mathrm{g}$ protein was loaded onto sodium dodecyl sulfate polyacrylamide gel electrophoresis gels $(8 \%)$, electrophoresed at $200 \mathrm{~V}$ for $2 \mathrm{~h}$ and subsequently transferred onto Immobilon ${ }^{\circledR}$-P polyvinylidene difluoride membranes (EMD Millipore, Billerica, MA, USA). The membranes were then incubated with the primary antibodies against HER2 (rabbit polyclonal; catalog no., ab58616; dilution, 1:150; Abcam, Cambridge, MA, USA) and $\beta$-actin (mouse monoclonal; catalog no., AA128; dilution, 1:1,000; Beyotime Institute of Biotechnology, Shanghai, China), followed by incubation with the appropriate goat monoclonal anti-rabbit and rabbit polyclonal anti-mouse secondary antibodies conjugated with alkaline phosphatase (catalog nos., A0208 and A0216, respectively; dilution, 1:1,000; Beyotime Institute of Biotechnology). Enhanced chemiluminescence chromogenic exposure and X-ray exposure were used for visualization.

Cell inhibition assay. Cell inhibition was analyzed using Cell Counting Assay kit-8 (CCK-8; Dojindo Molecular Technologies, Inc., Gaithersburg, MD, USA) according to the manufacturer's protocols. Briefly, the cell suspension was seeded in 96 -well plates at a density of $5 \times 10^{3}$ cells, with varying concentrations of cisplatin (DDP) added (0.312, 0.625, 1.25, $2.5,5,20,40$ and $20 \mu \mathrm{g} / \mathrm{ml}$ ), and cultured for $24 \mathrm{~h}$. A total of $10 \mu \mathrm{l}$ WST-8 was added to each well. The cells were incubated at $37^{\circ} \mathrm{C}$ for $1 \mathrm{~h}$ with $5 \% \mathrm{CO}_{2}$. An automatic microplate reader (Multiskan $^{\mathrm{TM}}$ MK3 Microplate Photometer; Thermo Fisher Scientific, Inc.) measured the absorbance (A) of each well at $450 \mathrm{~nm}$. The inhibition rate was calculated using the following equation: Inhibition rate $=\left(1-\mathrm{A}_{\text {dosing group }} / \mathrm{A}_{\text {cell control group }}\right) \times 100$.

Tumorigenicity assay in nude mice. Thymus-null BALB/c nude mice (female, 3-4 weeks old) were purchased from the Experimental Animal Center of Lake Hayes (Shanghai, China). All animal procedures were performed according to approved protocols and were in accordance with recommendations for the proper use and care of laboratory animals at the Experimental Animal Center of Soochow University (Suzhou, China). A total of $100 \mu 1$ of CON group and KD group cell solutions $\left(\sim 4 \times 10^{6}\right.$ cells $)$ were seeded in 25 nude mice subcutaneously, near the right armpit, to form three groups: The CON, CON + DDP and KD + DDP groups. The transplanted tumor diameters of the five nude mice were $\sim 5 \mathrm{~mm}$. The CON + DDP and KD + DDP mice groups were intravenously injected with DDP at a dose of $5 \mathrm{mg} / \mathrm{kg}$, once per week, over a period of 4 weeks. Tumor diameter was measured using Vernier calipers, and was used to calculate the tumor volume as $0.5 \mathrm{ab}^{2}$ (with a and $\mathrm{b}$ as the long and short diameters, respectively). At the end of the experiment, the mice were sacrificed by cervical dislocation. The tumors were excised, and immunohistochemical detection of HER2 protein expression and hematoxylin and eosin staining of tumor tissue was performed. 
A
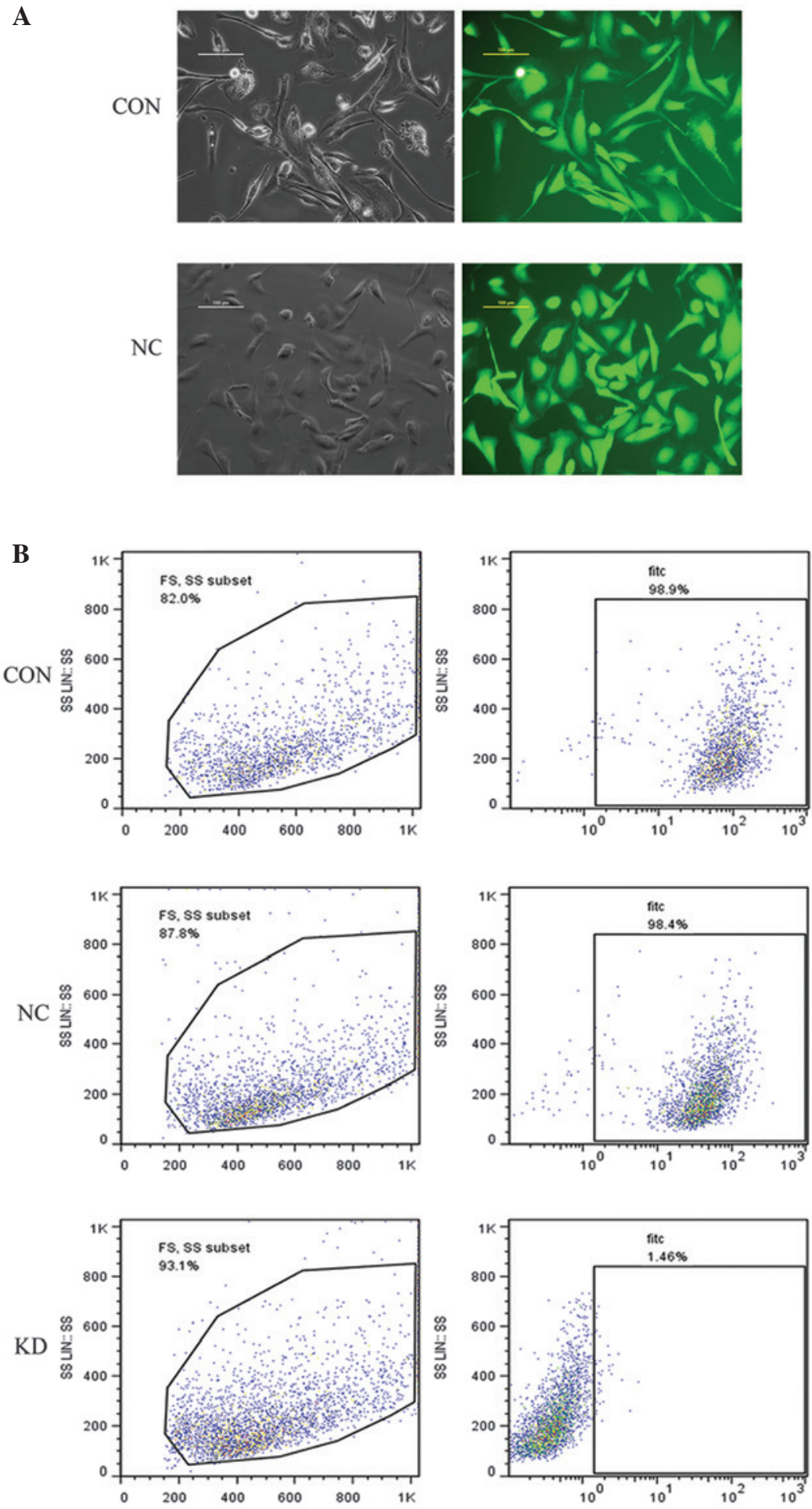

Figure 1. (A) Fluorescence expression of SKOV3 cell transfection with the lentiviral-mediated HER2-shRNA. Magnification, x200. (B) Fluorescent flow cytometry detected fluorescence expression of SKOV3 cell transfection with lentiviral-mediated HER2-shRNA. CON, blank control; NC, negative control; KD, SKOV3 RNA interference cell line; shRNA, small hairpin RNA; FITC, fluorescein isothiocyanate; SS, side scatter; FS, forward scatter; HER2, human epidermal growth factor receptor 2 .

Statistical analysis. All data are presented as the mean \pm standard deviation. Statistical significance was determined by conducting analysis of variance or $\chi^{2}$ tests, using SPSS software, version 17.0 (SPSS, Inc., Chicago, IL, USA). $\mathrm{P}<0.05$ was considered to indicate a statistically significant difference.

\section{Results}

Stable expression of HER2-shRNA in the SKOV3 and $N C$ cells. Lentiviral vector-infected SKOV3 cells were selected with puromycin for 2 weeks. Fluorescence microscopy (Fig. 1A) and flow cytometry (Fig. 1B) determined that 
A

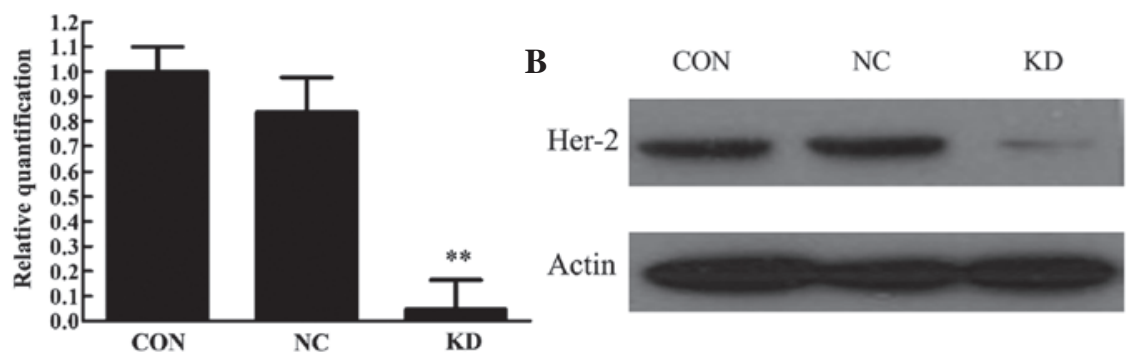

Figure 2. (A) Expression of HER-2 mRNA, as detected by reverse transcription-quantitative polymerase chain reaction following transfection with the lentiviral-mediated HER2-small hairpin RNA. Differences in the expression of HER-2 mRNA between the KD and NC and CON groups were significant $(\mathrm{P}<0.01 ; \mathrm{n}=3)$. ${ }^{* * *} \mathrm{P}<0.01$. (B) Western blot analysis to detect HER2 and actin protein expression in the SKOV3 cells. HER2 expression was decreased in the KD group when compared with the $\mathrm{CON}$ and $\mathrm{NC}$ groups $(\mathrm{P}<0.01 ; \mathrm{n}=3)$. CON, blank control; $\mathrm{NC}$, negative control; KD, SKOV3 RNA interference cell line; HER2, human epidermal growth factor receptor 2.

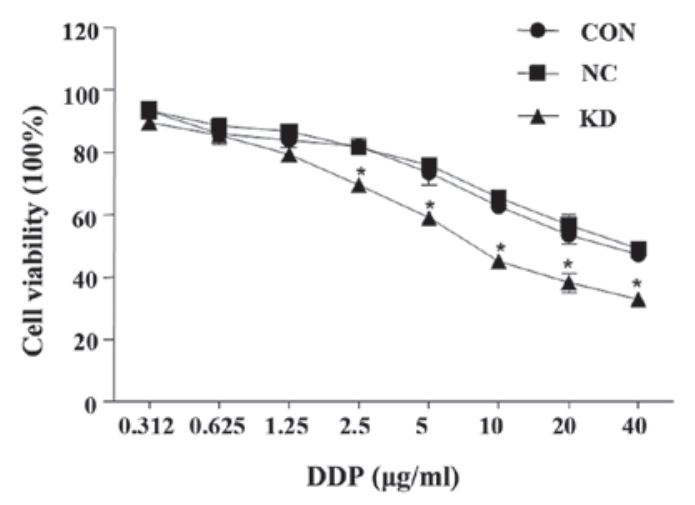

Figure 3. Cell viability of the SKOV3 cells following exposure to cisplatin at different concentrations, assessed by the cell counting kit- 8 assay. KD group viability decreased significantly when compared with the $\mathrm{NC}$ and $\mathrm{CON}$ groups $(\mathrm{P}<0.05 ; \mathrm{n}=3)$. ${ }^{*} \mathrm{P}<0.05$. $\mathrm{CON}$, blank control; $\mathrm{NC}$, negative control; KD, SKOV3 RNA interference cell line; DPP, cisplatin.

the infection efficiency of the NC and KD groups was $>98 \%$. The results demonstrate the successful establishment of stable HER2-shRNA and NC expression in the SKOV3 cell lines.

Stable expression of HER2-shRNA in the SKOV3 cells downregulates the HER2 gene. The relative mRNA content (HER2/ $\beta$-actin) in the CON, NC and KD cells was 1.000 , 0.837 and 0.045 , respectively. HER 2 mRNA levels in the KD group compared with the $\mathrm{CON}$ and $\mathrm{NC}$ group were lower, and the difference was statistically significant $(\mathrm{P}<0.01)$ (Fig. 2A). Western blot analysis of HER 2 protein expression following transfection of the three cell groups delivered results consistent with the mRNA levels (Fig. 2B).

Chemosensitivity to DDP in the SKOV3 cells. Following RNAi, chemosensitivity to DDP in the SKOV3 cells increased. With increasing concentrations of DDP administered, the proliferation rate of all three cell groups decreased; however, the decrease in the KD cell proliferation curve was more significant compared with the $\mathrm{NC}$ and $\mathrm{CON}$ groups $(\mathrm{P}<0.01$; Fig. 3).

HER2 silencing inhibits ovarian cancer growth in vivo. The results demonstrated that the tumor volume in the KD + DDP group was significantly less than that of the $\mathrm{CON}$ and $\mathrm{CON}+$ DDP groups $(\mathrm{P}<0.05)$, therefore indicating

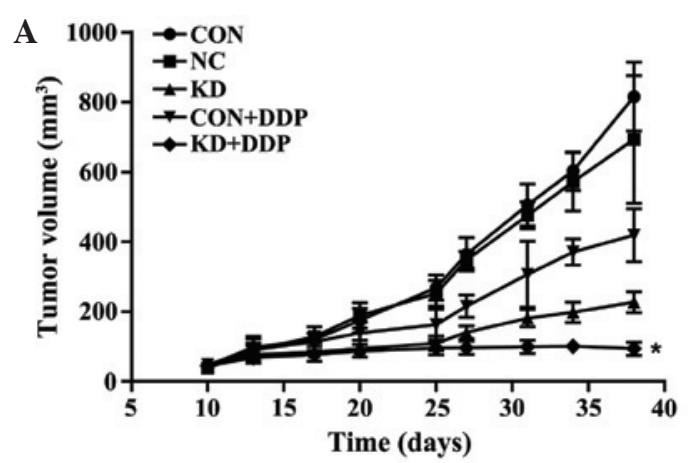

B CON NC KD CON+DDP KD+DDP

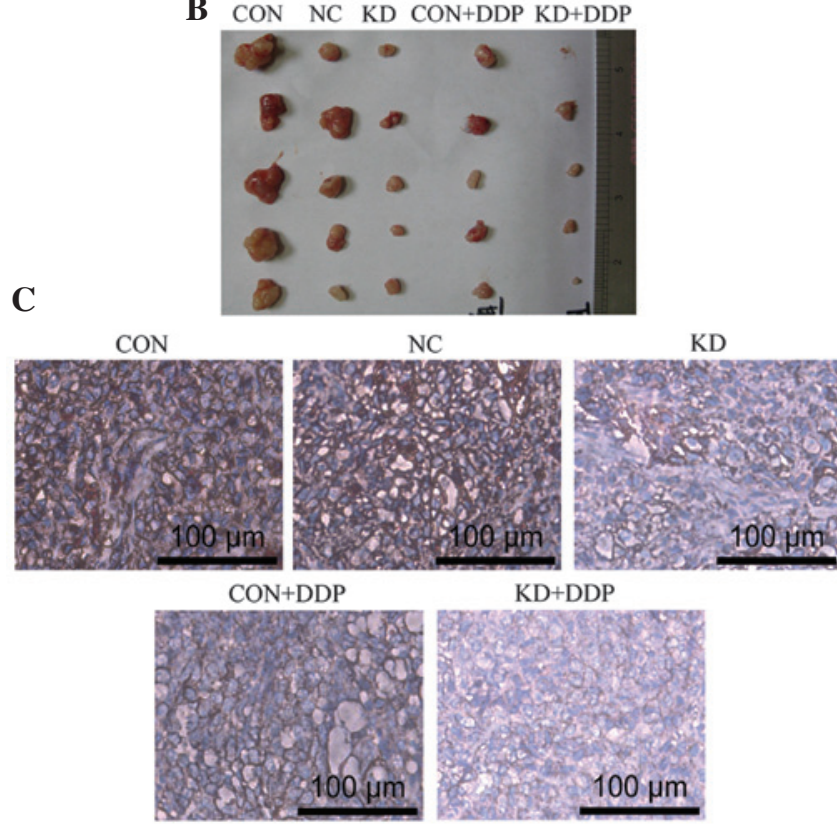

Figure 4. (A) Significant differences in tumor suppression in mice inoculated with the lentiviral-mediated HER2-shRNA SKOV3 cells and treated with DDP compared with the other four groups ( $\mathrm{P}<0.05 ; \mathrm{n}=5$ ). (B) Images of the tumors of the five groups. (C) Immunohistochemistry images showing significant differences in tumor suppression in mice inoculated with the lentiviral-mediated HER2-shRNA SKOV3 cells and treated with DDP, compared with the other four groups $(\mathrm{P}<0.05 ; \mathrm{n}=5)$. CON, blank control; $\mathrm{NC}$, negative control; $\mathrm{KD}$, SKOV3 RNA interference cell line; DPP, cisplatin; HER2, human epidermal growth factor receptor 2; shRNA, small hairpin RNA.

that DDP significantly inhibited the tumor growth rate. Lentivirus-mediated HER2-shRNA increased the sensitivity of the ovarian cancer xenografts to DDP (Fig. 4A and B). 
Immunohistochemical staining in the SKOV3 cells. Immunohistochemical staining of the CON group revealed that the area surrounding the nucleus was a deep brown color, showing positive staining for HER2. In the CON + DDP group, there were fewer of the brown particles compared with the CON group, showing weakly positive staining $(\mathrm{P}<0.05)$. The KD + DDP group had a significantly decreased number of brown particles, indicating that HER2 protein expression was significantly reduced (Fig. 4C).

\section{Discussion}

The treatment of ovarian cancer remains a challenge for clinicians (18). Despite the introduction of platinum-based chemotherapy and its improvement of the ovarian cancer patient survival rates, the long-term survival of patients remains low due to tumor recurrence and chemoresistance (19). Resistance to chemotherapeutics has been studied thoroughly and a number of mechanisms as to how this may occur have been proposed. A potential molecular marker that may improve the prediction of the patient response to a given treatment is HER2 overexpression, which affects the growth, proliferation, invasion and metastasis of cells (20). HER2 is the predominant cause of gynecological cancer mortality, and it has been demonstrated in vitro that the reduction of HER 2 expression, by antisense or siRNA, resulted in the inhibition of growth and the initiation of apoptosis in HER2 ${ }^{+}$breast and ovarian cancer cells $(4,21,22)$. Despite chemotherapeutic agents such as trastuzumab benefiting a large number of $\mathrm{HER}^{+}$patients, the development of drug resistance and toxic side effects may compromise the therapeutic effect (9).

In the present study, ovarian carcinoma SKOV3 cells were utilized as a model to analyze the effect of HER 2 expression and signaling levels on DDP sensitivity. RNAi was used to produce stable cell lines and the inhibition of HER2 gene expression was detected following the inhibition of the HER2 gene; furthermore, SKOV3 cell chemosensitivity to DDP was significantly enhanced. In vivo experiments demonstrated that the tumor volume in the KD + DDP group was significantly smaller than that of the other four groups. Tumor tissue immunohistochemistry indicated that the HER2 protein expression in the KD + DDP group was significantly lower than that in the other two groups, suggesting that lentiviral vector-mediated HER2-shRNA increases cell sensitivity to DDP in ovarian cancer. Such results provide a theoretical basis for novel therapies for chemotherapy-resistant ovarian cancers.

In the current study, lentiviral-mediated shRNA expression vectors, as compared with plasmid-mediated siRNA, were stably expressed for a long period of time, and the preparation of cell lines stably expressing shRNA was the most effective means for the in vivo experiments. The use of lentivirus in an organism may induce gene mutations and function as a potential biological hazard; therefore, it is important to demonstrate that they can be safely applied to the human body (23). As technology continues to advance, the use of RNAi may become an important means for future cancer gene therapy.

In conclusion, the present study demonstrated that HER2 serves an important role in the chemoresistance of ovarian cancer. However, further clarification of its functional characterization is required. The results of the present study provide support for the possible development of a novel gene therapy targeting HER2, ultimately aiming to prevent chemoresistance in human ovarian cancer.

\section{References}

1. Siegel R, Naishadham D and Jemal A: Cancer statistics, 2013. CA Cancer J Clin 63: 11-30, 2013.

2. Salani R, Santillan A, Zahurak ML, et al: Secondary cytoreductive surgery for localized, recurrent epithelial ovarian cancer: Analysis of prognostic factors and survival outcome. Cancer 109: 685-691, 2007.

3. Helm CW: Current status and future directions of cytoreductive surgery and hyperthermic intraperitoneal chemotherapy in the treatment of ovarian cancer. Surg Oncol Clin N Am 21: 645-663, 2012.

4. Lu YM, Rong ML, Shang C, et al: Suppression of HER-2 via siRNA promotes apoptosis and decreases metastatic potential of SKOV3 human ovarian carcinoma cells. Oncol Rep 29: 1133-1139, 2013.

5. Baselga $J$ and Arteaga CL: Critical update and emerging trends in epidermal growth factor receptor targeting in cancer. J Clin Oncol 23: 2445-2459, 2005.

6. Camilleri-Broët S, Hardy-Bessard AC, Le Tourneau A, Paraiso D, Levrel O, Leduc B, Bain S, Orfeuvre H, Audouin J and Pujade-Lauraine E; GINECO group: HER-2 overexpression is an independent marker of poor prognosis of advanced primary ovarian carcinoma: A multicenter study of the GINECO group. Ann Oncol 15: 104-112, 2004.

7. Lanitis E, Dangaj D, Hagemann IS, et al: Primary human ovarian epithelial cancer cells broadly express HER2 at immunologically-detectable levels. PLoS One 7: e49829, 2012.

8. Neve RM, Lane HA and Hynes NE: The role of overexpressed HER2 in transformation. Ann Oncol 12 (Suppl 1): S9-S13, 2001.

9. Tai W, Mahato R and Cheng K: The role of HER2 in cancer therapy and targeted drug delivery. J Control Release 146: 264-275, 2010.

10. Yu D and Hung MC: Role of erbB2 in breast cancer chemosensitivity. BioEssays 22: 673-680, 2000.

11. Berchuck A, Kamel A, Whitaker R, et al: Overexpression of HER-2/neu is associated with poor survival in advanced epithelial ovarian cancer. Cancer Res 50: 4087-4091, 1990.

12. Tan DS, Ang JE and Kaye SB: Ovarian cancer: Can we reverse drug resistance? Adv Exp Med Biol 622: 153-167, 2008.

13. Fire A, Xu S, Montgomery MK, et al: Potent and specific genetic interference by double-stranded RNA in Caenorhabditis elegans. Nature 391: 806-811, 1998.

14. Draz MS, Fang BA, Zhang P, et al: Nanoparticle-mediated systemic delivery of siRNA for treatment of cancers and viral infections. Theranostics 4: 872-892, 2014.

15. Meister $\mathrm{G}$ and Tuschl T: Mechanisms of gene silencing by double-stranded RNA. Nature 431: 343-349, 2004.

16. Brodersen P, Sakvarelidze-Achard L; Bruun-Rasmussen M, et al: Widespread translational inhibition by plant miRNAs and siRNAs. Science 320: 1185-1190, 2008.

17. Tuschl T: Expanding small RNA interference. Nat Biotechnol 20: 446-448, 2002.

18. Bristow RE, Tomacruz RS, Armstrong DK, Trimble EL and Montz FJ: Survival effect of maximal cytoreductive surgery for advanced ovarian carcinoma during the platinum era: A meta-analysis. J Clin Oncol 20: 1248-1259, 2002.

19. Ali AY, Farrand L, Kim JY, et al: Molecular determinants of ovarian cancer chemoresistance: New insights into an old conundrum. Ann N Y Acad Sci 1271: 58-67, 2012.

20. Takehana T, Kunitomo K, Suzuki S, et al: Expression of epidermal growth factor receptor in gastric carcinomas. Clin Gastroenterol Hepatol 1: 438-445, 2003.

21. Yang G, Cai KQ, Thompson-Lanza JA, Bast RC Jr and Liu J: Inhibition of breast and ovarian tumor growth through multiple signaling pathways by using retrovirus-mediated small interfering RNA against Her-2/neu gene expression. J Biol Chem 279: 4339-4345, 2004.

22. Roh H, Pippin J and Drebin JA: Down-regulation of HER2/neu expression induces apoptosis in human cancer cells that overexpress HER2/neu. Cancer Res 60: 560-565, 2000.

23. Dullaers M, Van Meirvenne S, Heirman C, et al: Induction of effective antitumor immunity by direct in vivo administration of lentiviral vectors. Gene Ther 13: 630-640, 2005. 